\title{
E2A-positive gastric MALT lymphoma has weaker plasmacytoid infiltrates and stronger expression of the memory B-cell-associated miR-223: possible correlation with stage and treatment response
}

\author{
Ting-Yun Liu ${ }^{1,4}$, Shee-Uan Chen ${ }^{2,4}$, Sung-Hsin Kuo ${ }^{3}$, Ann-Lii Cheng ${ }^{3}$ and Chung-Wu Lin ${ }^{1}$ \\ ${ }^{1}$ Department of Pathology, National Taiwan University Hospital, National Taiwan University College of \\ Medicine, Taipei, Taiwan, ROC; ${ }^{2}$ Department of Gynecology and Obstetrics, National Taiwan University \\ Hospital, National Taiwan University College of Medicine, Taipei, Taiwan, ROC and ${ }^{3}$ Department of \\ Oncology, National Taiwan University Hospital, National Taiwan University College of Medicine, Taipei, \\ Taiwan, $R O C$
}

\begin{abstract}
Extranodal marginal-zone lymphoma of mucosa-associated lymphoid tissue of the stomach (gastric MALT lymphoma) is derived from memory $B$ cells of the marginal zone. Normal memory $B$ cells do not express markers of germinal-center B cells, such as E2A (immunoglobulin enhancer-binding factor E12/E47), B-cell chronic lymphocytic leukemia/lymphoma $6(B C L 6)$, or activation-induced cytidine deaminase $(A I D)$. E2A is a transcription factor that induces somatic hypermutations and blocks plasma cell differentiation. In 50 stage- $I_{E} / I_{E 1}$ gastric MALT lymphomas, we confirmed that all cases were BCL6 IAID , but a subset $(50 \%, 25 / 50)$ was $E 2 A^{+}$. As $E 2 A^{-}$and $E 2 A^{+}$gastric MALT lymphomas had similar numbers of somatic hypermutations without intraclonal variations, which implied an origin from memory $B$ cells, the expression of E2A was best regarded as a marker of aberrant follicular differentiation. Although the status of somatic hypermutation was not affected by $E 2 A, E 2 A^{+}$gastric MALT lymphoma showed less plasmacytoid infiltrates and higher expressions of miRNA-223, a microRNA associated with memory B cells. Clinically, E2A ${ }^{+}$gastric MALT lymphomas were more likely to spread to perigastric lymph nodes and were less responsive to Helicobacter eradication therapy than were E2A ${ }^{-}$gastric MALT lymphomas. Taken together, aberrant E2A expression is a diagnostic feature of a subtype of gastric MALT lymphoma with weaker plasmacytoid infiltrates and stronger miR-223 expression. A prospective study would be necessary to verify the association between E2A expression and a poor response to Helicobacter eradication therapy.

Modern Pathology (2010) 23, 1507-1517; doi:10.1038/modpathol.2010.139; published online 27 August 2010
\end{abstract}

Keywords: E2A; gastric MALT lymphoma; Helicobacter pylori eradication therapy; memory B cell; miR-223; plasma cell

Extranodal marginal-zone lymphoma of mucosaassociated lymphoid tissue of the stomach (gastric MALT lymphoma) is a Helicobacter pylori

Correspondence: Dr C-W Lin, Department of Pathology, National Taiwan University College of Medicine, 1-1 Jen-Ai Road, Taipei 10016, Taiwan.

E-mail: chungwulin@yahoo.com

${ }^{4}$ These authors contributed equally to this work.

Received 14 April 2010; revised 1 June 2010; accepted 7 June 2010; published online 27 August 2010 infection-associated lymphoma that depends on the stimulus of Helicobacter for growth. ${ }^{1}$ About $70 \%$ of gastric MALT lymphomas can be cured by $H$. pylori eradication therapy with antibiotics, ${ }^{2}$ but cases with API2-MALT1 fusion because of $\mathrm{t}(11 ; 18)(\mathrm{q} 21 ; \mathrm{q} 21)$ or with nuclear expression of $N F \kappa B$ or B-cell chronic lymphocytic leukemia/lymphoma 10 (BCL10) are unresponsive. . $^{3,4}$

From the pathologists' perspective, gastric MALT lymphoma is an extranodal marginal-zone lymphoma. 
In the current WHO classification, mantle cell lymphoma, follicular lymphoma, and marginal-zone lymphoma are distinct entities representing malignant transformations of mantle cells, germinalcenter B cells, or memory B cells, respectively. ${ }^{5}$ However, morphologic, phenotypic, and genetic overlaps among these lymphomas have not been well documented. Interestingly, most reports showed aberrant marginal-zone differentiation in follicular lymphoma, ${ }^{6,7}$ but not the opposite phenomenon of aberrant follicular differentiation in marginal-zone lymphoma. In this study, we investigated the expression patterns of transcription factors, the status of somatic hypermutation, and microRNA (miRNA) signatures to detect such aberrant follicular differentiation in gastric MALT lymphoma.

Aberrant follicular differentiation could be identified through comparisons between gastric MALT lymphoma and its normal counterparts, memory B cells. ${ }^{8}$ Memory B cells are derived from naive B cells through either a T-independent marginal-zone pathway or a T-dependent follicular pathway. ${ }^{9}$ E2A (immunoglobulin enhancer-binding factor E12/E47) is a transcription factor found on germinal-center $\mathrm{B}$ cells. E2A may stimulate somatic hypermutation, which is characteristic of the T-dependent follicular pathway and favors the development of germinalcenter B cells. ${ }^{10,11}$ Furthermore, down-regulation of $E 2 A$ is required for differentiation of germinalcenter B cells into plasma cells. ${ }^{12}$ Therefore, expression of E2A on gastric MALT lymphoma might be a potential marker of aberrant follicular differentiation, and might be associated with either an increase in somatic hypermutations or a block in terminal plasma cell differentiation.

$B C L 6$, a transcription factor for follicle formation, ${ }^{13}$ and activation-induced cytidine deaminase $(A I D)$, an enzyme inducing somatic hypermutation, ${ }^{, 4,15}$ are both important players in the development of germinal-center B cells. In contrast, T-cell leukemia/lymphoma 1A (TCL1), ${ }^{16,17}$ whose functions are still unclear, is a marker for B cells of the mantle zone. Abnormal patterns of these markers might reveal aberrant differentiation of gastric MALT lymphoma as well.

Aberrant follicular differentiation in gastric MALT lymphoma might be reflected in the status of somatic hypermutation, which has been used widely to characterize B-cell lymphomas. ${ }^{18}$ For example, follicular lymphomas derived from germinal-center B cells typically have somatic hypermutations with intraclonal variations, whereas gastric MALT lymphomas from memory B cells usually have somatic hypermutations without intraclonal variations. However, unusual cases of gastric MALT lymphomas with somatic hypermutations and intraclonal variations were reported. ${ }^{19,20}$ As E2A stimulates somatic hypermutations, aberrant expression of E2A and abnormal patterns of somatic hypermutations might be associated.
Finally, germinal-center B cells and memory B cells have distinct miRNA patterns, and they could be distinguished with a panel of 39 miRNAs. ${ }^{21}$ From this panel, it was reported that a minimal subset of six miRNAs was sufficient to distinguish germinalcenter B cells from memory B cells: miR-17-5p, miR20b, miR-93, miR-28, and miR-181b were markers of germinal-center b cells, whereas miR-223 was a marker of memory B cells. ${ }^{22}$

In this retrospective study of 50 gastric MALT lymphomas that had received $H$. pylori eradication therapy and were monitored by multiple endoscopic biopsies, we investigated the expression patterns of E2A, BCL6, AID, TCL1, somatic hypermutations, and miRNAs to identify aberrant follicular differentiation and the biological and clinical significance of such aberrant differentiation.

\section{Materials and methods}

\section{Patients and Samples}

From the archives at the Pathology Department of National Taiwan University Hospital, 50 stage- $\mathrm{I}_{\mathrm{E}}$ or $\mathrm{II}_{\mathrm{E} 1}$ Helicobacter-positive gastric MALT lymphomas were selected for this study. All cases were treated with $H$. pylori eradication therapy and followed by multiple endoscopic biopsies every 6-12 weeks. The cases were selected on a consecutive base, but cases with inadequate materials for nucleic acid extraction were excluded. Stage- $\mathrm{I}_{\mathrm{E}}$ gastric MALT lymphomas were limited to the stomach, and stage- $\mathrm{II}_{\mathrm{E} 1}$ gastric MALT lymphomas involved both the stomach and perigastric lymph nodes. The diagnosis of gastric MALT lymphoma was based on the WHO criteria, characterized by centrocyte-like infiltrates and lymphoepithelial lesions, without clusters or sheets of large cells. The initial biopsies had a Wotherspoon score of 4 or $5 .^{2,23}$ The diagnosis of Helicobacter infection was based on a combination of morphology and the urease test on the biopsy specimen. Staging procedures and $H$. pylori eradication therapy were performed according to established protocols. ${ }^{4}$ At each follow-up examination, six biopsies were taken from the tumors for evaluation. Responsive cases were defined as having a histologic remission with a Wotherspoon score of 0 , 1 , or 2 within 6 months. ${ }^{2,23}$

\section{Immunohistochemistry}

Immunoperoxidase stains on formalin-fixed, paraffin-embedded tissue sections of gastric MALT lymphomas, chronic gastritis, and FLs were performed with an automatic immunostainer (Benchmark, Ventana Medical System, Tucson, AZ). The following antibodies were used: $A I D$ (1:40, clone ZA001, Zymed Laboratories, South San Francisco, CA), BCL6 (1:20, clone P1F6 + PGB6P, Neomarker, Fremont, CA), E2A (1:200, polyclonal, Santa Cruz 
Biotech, Santa Cruz, CA), MUM1 (1:50, MUM1p, DAKO, Glostrup, Denmark), and TCL1 (1:200, clone 1-21, Zymed Laboratories). Antigen retrieval was performed in Tris buffer ( $\mathrm{pH} 8$ for $A I D, \mathrm{pH} 9$ for $B C L 6$, pH 6 for E2A, MUM1, and TCL1), and primary antibodies were applied to the slides at $37 \mathrm{C}$ for $45 \mathrm{~min}$. Biotin-conjugated secondary antibodies, peroxidase-conjugated streptavidin, and DAB were used sequentially to complete the reactions.

Immunoperoxiadse stains for $C D 138$ (1:100, MI15, DAKO) and CD68 (1:100, KP1, DAKO) were performed on formalin-fixed, paraffin-embedded tissue sections of gastric MALT lymphoma according to the vendor's recommendations.

\section{Amplification of the Immunoglobulin Heavy Chain Variable Region by PCRs}

DNA from formalin-fixed, paraffin-embedded tissue blocks was amplified by a forward framework 1 consensus primer, 5'-AGGTGCAGCTG(G/C)(A/T)G(G/C) AGTC(G/A/T)GG-3', and a reverse junctional consensus primer, 5' -CTTACCTGAGGAGACGGTGA-3' ${ }^{24}$ Sequences of the PCR products were analyzed with IMGT/V-quest at http://imgt.cines.fr. ${ }^{25} \mathrm{~V}$-D-J recombinations that were out-of-frame or in-frame with stop codons were excluded from analysis. For each case, a consensus sequence with an in-frame V-D-J recombination not interrupted by a stop codon was established. Total numbers of somatic hypermutations and the ratios of replacement to silent mutations in the framework regions and the complementarity determining regions were calculated. ${ }^{26,27}$ Deviations from the consensus were regarded as intraclonal variations. The numbers of deviations divided by the number of sequences were calculated. In four gastric MALT lymphomas, PCRs were repeated on different days, and identical sequences were obtained. Thus, the background mutations in our system were at most one mutation per sequence of the immunoglobulin heavy variable region, and they did not affect our sequence analyses.

\section{Quantitative RT-PCR for miRNAs}

Total RNAs were extracted from formalin-fixed, paraffin-embedded tissue blocks with the Trizol method. Quantification of miRNAs by a stem-loop RT-PCR was performed according to a published protocol with modification. ${ }^{28}$

For RT, the following miRNA-specific primers were used: $5^{\prime}-\mathrm{N}-\mathrm{ACTACC}-3^{\prime}(m i R-17-5 p), 5^{\prime}$-N-CTAC CT-3' (miR-20b), 5'-N-CTCAAT-3' (miR-28-5p), 5'-N-CT ACCT-3' (miR-93), 5'-N-ACCCAC-3' (miR-181), 5'-N-TG GGGT-3' (miR-223), and 5'-N-ACAAAAA TATGGAA CGCTT-3' ${ }^{\prime}(U 6)$, where N was 5'-GTCGTATCCATGGC AGGGTCCGAGGTA TTCGCCATGGATACGAC-3'. The RT mixture included $0.1 \mu \mathrm{M}$ miRNA-specific stemloop RT primer, $10 \mathrm{mM}$ dNTPs, $50 \mathrm{mM}$ Tris-HCl at pH 8.3, $75 \mathrm{mM} \mathrm{KCl,} 3 \mathrm{mM} \mathrm{MgCl}$, 0.1 M DTT, 100 units of reverse transcriptase, and $1 \mu \mathrm{g}$ RNA in a final volume of $20 \mu \mathrm{l}$. The mixture was incubated at $42^{\circ} \mathrm{C}$ for $80 \mathrm{~min}$.

For PCR, a universal reverse PCR primer, 5'-TGG CAGGGTCCGAGGT-3', and the following miRNAspecific forward primers were used: $5^{\prime}$-CAAAGT GCTTACAGTG-3' (miR-17-5p), 5'-CAAAGTGCTCA TAGTG-3' (miR-20b), 5'-AAGGAGCTCACAGTCT-3' (miR-28-5p), 5'-CAAAGTGCTGTTCGTG-3' (miR-93), $5^{\prime}$-AACATTCATTGCTGTC-3' (miR-181), 5'-TGTCAG TTTGTCAAAT-3' (miR-223), and 5'-GTGCTCGCTTC GGCAGC ACA-3' (U6). Real-time PCRs were performed in a $20 \mu \mathrm{l}$ reaction mixture that included $5 \mu \mathrm{l}$ diluted CDNA, $0.1 \mu \mathrm{M}$ miRNA-specific forward primer, $0.1 \mu \mathrm{M}$ universal reverse primer, and $10 \mu \mathrm{l}$ of SYBR Green PCR master mix (TOYOBO, Japan). A PCR cycle consisted of denaturation at $95^{\circ} \mathrm{C}$ for $15 \mathrm{~s}$ and annealing at $60^{\circ} \mathrm{C}$ for $1 \mathrm{~min}$. The PCR products, 54-58 bp for miRNAs and $140 \mathrm{bp}$ for U6, were confirmed by $8 \%$ acrylamide gel and sequencing. The differences between the threshold cycles of the miRNAs and U6 snRNA, dCT $\left(\mathrm{CT}_{\mathrm{U} 6}-\mathrm{CT}_{\text {miRNA }}\right)$, were calculated from the mean values of duplicate measurements. This definition of dCT implies a positive correlation between dCTs and miRNA levels.

\section{Statistical Analysis}

The nonparametric Mann-Whitney test or Fisher's test was used for comparison of continuous or categorical data, respectively.

\section{Results}

\section{E2A Was Expressed on the Follicular but not the Marginal-Zone Cells in Chronic Gastritis}

Immunoperoxidase stains on chronic gastritis with reactive follicles showed that TCL1 was expressed in the mantle zone, whereas BCL6, AID, and E2A were expressed in the follicles (Figure 1a-d). These results were consistent with reports on lymph nodes. ${ }^{10-17}$ The restricted expression of E2A in the follicles was also consistent with its function in inducing somatic hypermutations.

Gastric MALT Lymphomas Were Negative for TCL1, $B C L 6$, and $A I D$, but Strong E2A Expression in a Subset of Gastric MALT Lymphomas Suggested Aberrant Follicular Differentiation

Except for focal staining of TCL1, BCL6, or AID in the residual follicles that were trapped inside the lymphoma, the majority of lymphoma cells of all 50 gastric MALT lymphomas were negative for TCL1, BCL6, and AID (Figure 1f-h), consistent with a phenotype of memory $B$ cells of the marginal zone.

In contrast, the expression of E2A was more variable. A case of gastric MALT lymphoma with 
about $70 \% E 2 A^{+}$lymphoma cells was noted (Figure 1i). We found that 25 out of the 50 gastric MALT lymphomas in our series had $>40 \% E 2 A^{+}$ lymphoma cells. These cases were regarded as $E 2 A^{+}$.
As follicular lymphomas were $T C L 1^{-} / B C L 6^{+} / A I D^{+} /$ $\mathrm{E}_{2} \mathrm{~A}^{+}$(Figure $1 \mathrm{k}-\mathrm{n}$ ), the expression of E2A in a subset of gastric MALT lymphomas was suggestive of aberrant follicular differentiation.

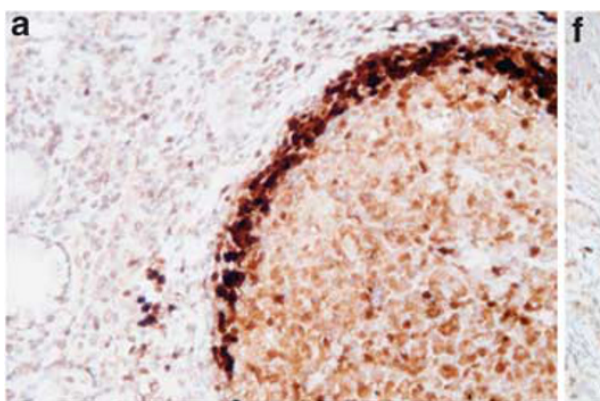

b
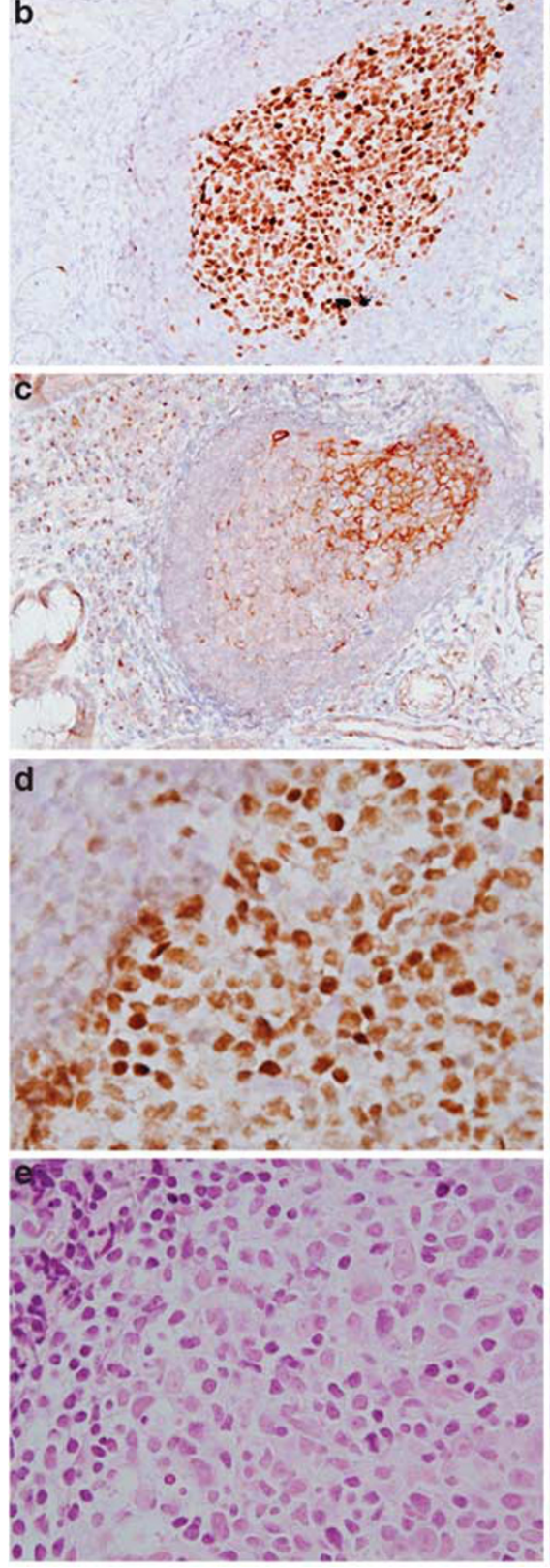

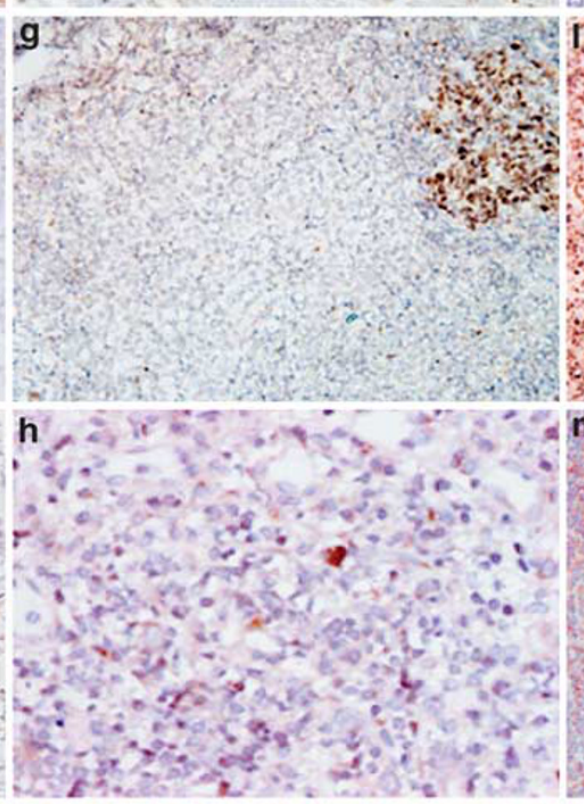

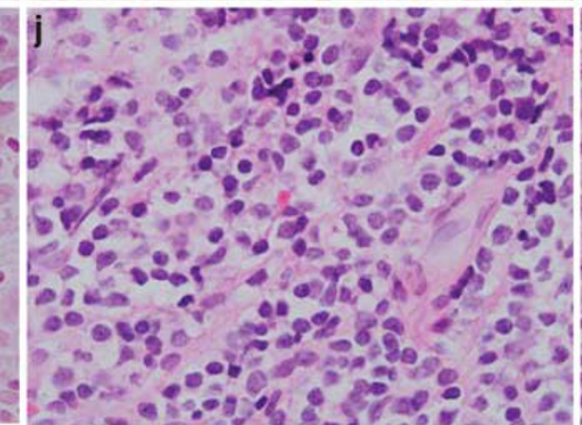

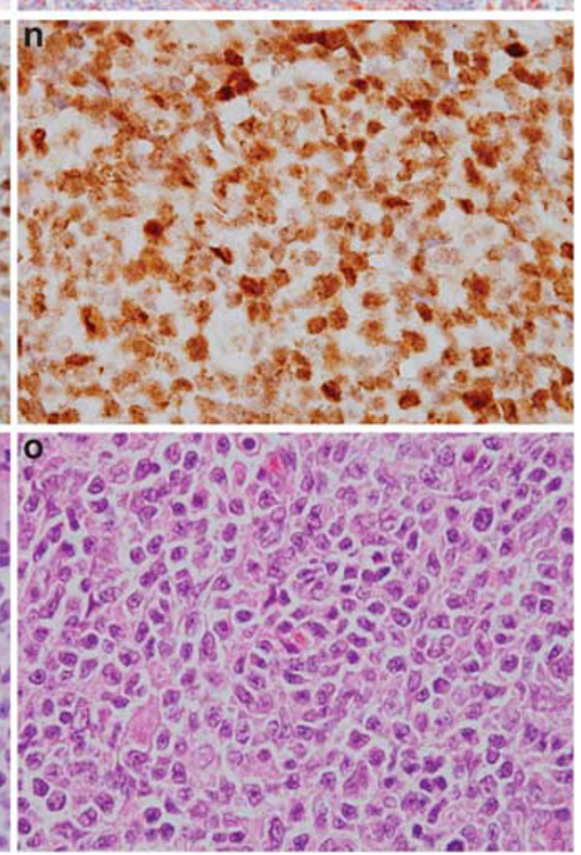


$E 2 A^{-}$and $E 2 A^{+}$Gastric MALT Lymphomas Had Similar Somatic Hypermutations without Intraclonal Variations, Consistent with an Origin from Memory B Cells

The immunoglobulin heavy chain variable regions were sequenced in $10 \mathrm{E} \mathrm{A}^{-}$and $10 \mathrm{E}^{-} \mathrm{A}^{+}$gastric MALT lymphomas. Although E2A could stimulate somatic hypermutations in germinal-center $B$ cells, the $E 2 A^{-}$and $E 2 A^{+}$groups had similar somatic hypermutations (18 vs 29, $P=0.31$; Figure 2a). The numbers of intraclonal variations were $<1$ (0.3 vs 0.4, $P=0.77$; Figure 2b). Thus, $E 2 A^{-}$and $E 2 A^{+}$ gastric MALT lymphomas had similar somatic
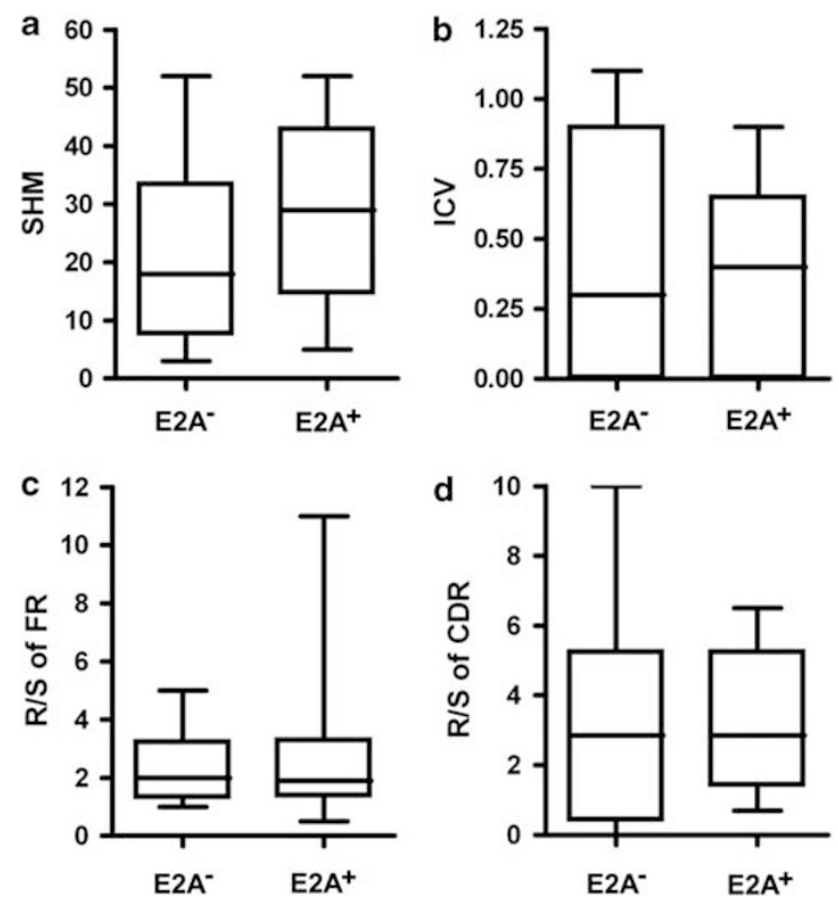

Figure $2 \mathrm{E}^{2} \mathrm{~A}^{-}$and $E 2 \mathrm{~A}^{+}$gastric MALT lymphomas had similar somatic hypermutations without intraclonal variations, consistent with an origin from memory B cells. Immunoglobulin heavy chain variable regions were sequenced in $10 \mathrm{E}^{-A^{-}}$and $10 \mathrm{E}^{2} \mathrm{~A}^{+}$ gastric MALT lymphomas. The medians for somatic hypermutations, intraclonal variations, and ratios of replacement to silent mutations were compared with the box-and-whisker plots. (a) Somatic hypermutations, 18 vs 29 at $P=0.31$; (b) Intraclonal variations, 0.3 vs 0.4 at $P=0.77$; (c) ratios of replacement to silent mutations in the framework regions, 2.0 vs 1.9 at $P=0.85$; (d) ratios of replacement to silent mutations in the complementarity determining regions, 2.9 vs 2.9 at $P=0.88$. hypermutations without intraclonal variations, consistent with an origin from memory B cells.

\section{$E 2 A^{-}$and $E 2 A^{+}$Gastric MALT Lymphomas Had Similar Ratios of Replacement to Silent Mutations}

In B-cell lymphomas, an increase in the ratios of replacement to silent mutations in the framework regions and the complementarity determining regions of the immunoglobulin genes has been used as indicators of antigen selection pressure that contributes to the development of the lymphomas. As gastric MALT lymphoma is driven by the stimulus of Helicobacter infection, we evaluated the ratios of replacement to silent mutations to see whether the ratios were correlated with the expression of $E 2 A{ }^{26,27}$ The ratios in the framework regions $(2.0$ vs 1.9, $P=0.85$; Figure 2c) and in the complementarity determining regions $(2.9$ vs 2.9 at $P=0.88$; Figure 2d) were similar.

Without significant differences in somatic hypermutations, intraclonal variations, or the ratios replacement to silent mutations between $E 2 A^{-}$and $E 2 A^{+}$ gastric MALT lymphomas, the biological effect of $E 2 A$ expression in gastric MALT lymphoma was not the induction of somatic hypermutations, ${ }^{10,11}$ but might be the prevention of plasma cell differentiation. ${ }^{12}$

\section{Aberrant E2A Expression Was Associated with Less Plasmacytoid Infiltrates}

Gastric MALT lymphomas, which are mainly composed of centrocyte-like lymphoma cells, frequently have plasma cell differentiation. As one of the biological effects of $E 2 A$ is to block plasma cell differentiation, aberrant $E 2 A$ expression in gastric MALT lymphomas might be associated with decreased numbers of plasma cells.

During the transition from germinal-center B cells to plasma cells, E2A was down-regulated and another transcription factor, MUM1, was up-regulated concurrently. ${ }^{12}$ Therefore, immunohistochemical stain of the plasma cell marker MUM1 was performed (Figure 3). The presence of $M U M 1^{+}$cells, either inside the tumor or adjacent to the foveolar glands outside the tumor, was determined. We found that 12 out of $25 E 2 A^{-}$cases and 4 out of

Figure 1 Distinct expression patterns of TCL1, AID, BCL6, and E2A were found in chronic gastritis, gastric MALT lymphoma, and follicular lymphoma. From top to bottom: immunostains for TCL1, BCL6, AID, and E2A, and H/E stain; from right to left: chronic gastritis, gastric MALT lymphoma, and follicular lymphoma. For chronic gastritis, TCL1 stained the mantle zone strongly and the follicular zone weakly (a), and $B C L 6(\mathbf{b}), A I D(\mathbf{c})$, and $E 2 A(\mathbf{d})$ all stained the follicular zone. Note that most lymphocytes in the mantle or marginal zone were negative for BCL6 (b), $A I D(\mathbf{c})$, and $E 2 A$ (d). The corresponding H/E section showed a follicle rimmed by the mantle zone (e). For gastric MALT lymphoma, TCL1, BCL6, and $A I D(\mathbf{f}-\mathbf{h})$ were negative, but the expression of $E 2 A$ (i) could be positive, consistent with a marginal-zone origin with aberrant follicular differentiation. TCL1 stained scattered lymphoma cells (f), BCL6 stained a residual follicle (g), and AID was negative (h). Note that most lymphoma cells were negative for these three markers. However, E2A stained about $70 \%$ of the cells in the marginal zone (i). The corresponding H/E section showed monocytoid differentiation of the lymphoma cells (j). For follicular lymphoma, TCL1 was negative (k), and BCL6, AID, and E2A (l-n) were positive. Note that the residual mantle zone (k) was $T C L 1^{+}$. The corresponding H/E section was shown (o). Immunoperoxidase stains on formalin-fixed, paraffinembedded tissue sections, $\times 400(\mathbf{a}-\mathbf{c}, \mathbf{f}-\mathbf{h}, \mathbf{k}-\mathbf{m})$ or $\times 1000(\mathbf{d}-\mathbf{e}, \mathbf{i}-\mathbf{j}, \mathbf{n}-\mathbf{o})$. 


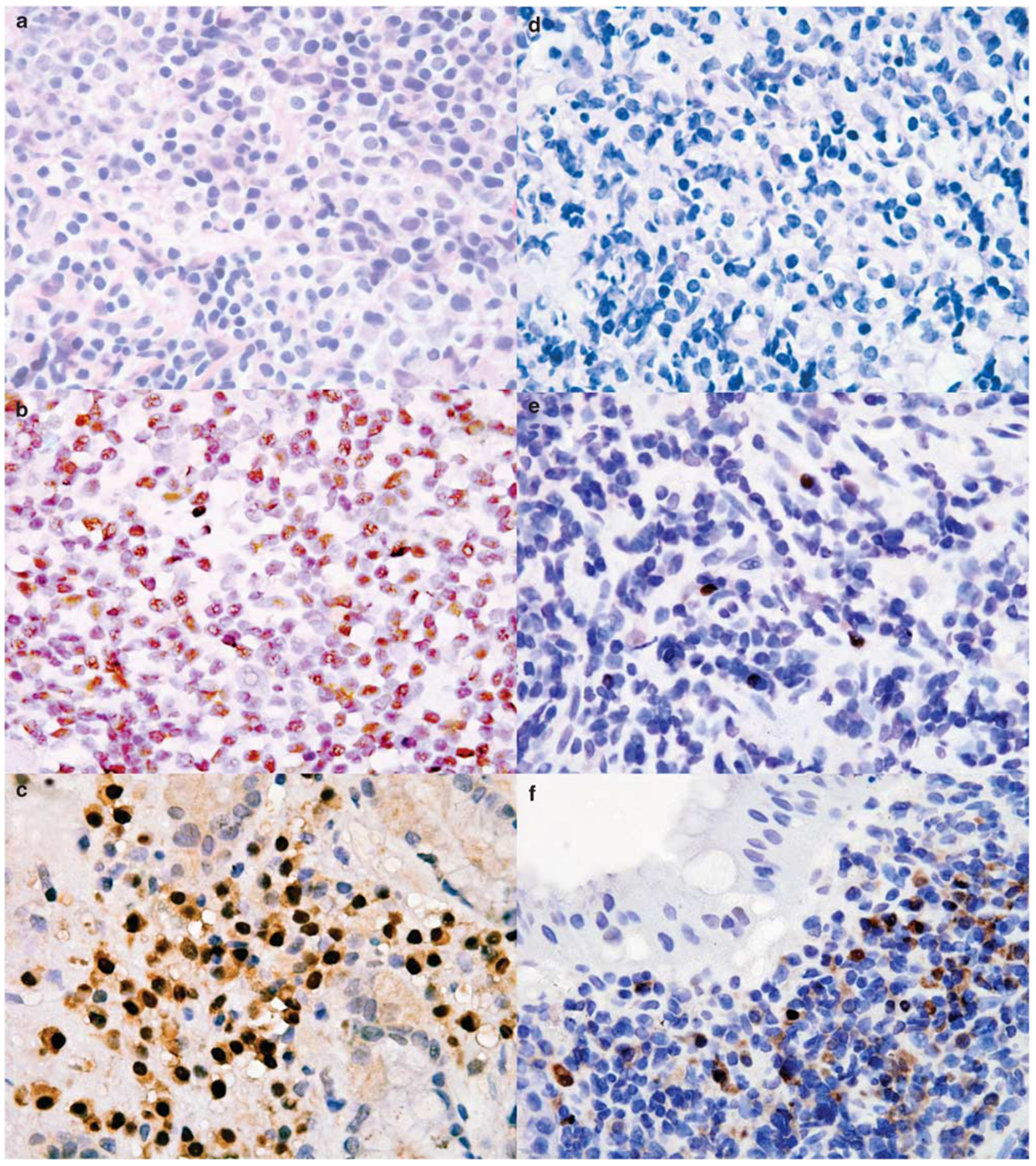

Figure $3 \mathrm{E}^{2} \mathrm{~A}^{+}$gastric MALT lymphomas had less plasmacytoid infiltrates. $E 2 A^{-}$gastric MALT lymphomas (a) often had $M U M 1^{+}$cells both inside the tumor (b) and next to the foveolar glands at the periphery of the tumor (c). In contrast, E2 $A^{+}$gastric MALT lymphomas (d) did not show $M U M 1^{+}$cells inside the tumor (e) or next to the foveolar glands (f). H/E (a, d) and immunoperoxidase (b, c, e, f) stains were performed on formalin-fixed, paraffin-embedded tissue sections, all at $\times 1000$.

$25 \mathrm{E}_{2} \mathrm{~A}^{+}$cases had plasmacytoid infiltrates $(48 \%$ vs $16 \%, P=0.02$ ). Immunostains for $C D 138$, another marker for plasma cells, confirmed the presence of plasma cells in 6 of $10 E 2 A^{-}$cases and 2 of 10 $E 2 A^{+}$cases.
These data supported that aberrant E2A expression was associated with a block in plasma cell differentiation, although a definitive conclusion would require isolation of the $M U M 1^{+}$cells to establish clonality by flow cytometry. 
$\mathrm{E2A}^{+}$Gastric MALT Lymphomas Had Higher Levels of miR-223, a Memory B-cell-associated miRNA, than Did E2A- Gastric MALT Lymphomas

The expression profile of six miRNAs was determined, including five germinal-center B-cell-associated miRNAs (miR-28-5p, miR-93, miR-17-5p, miR-20b, and miR-181b), and a memory B-cellassociated miRNA (miR-223). This panel was chosen from the 39 miRNAs reported to separate germinal-center B cells from memory B cells, and included all six miRNAs that were sufficient for separation of germinal-center B cells from memory B cells. ${ }^{21,22}$

The miRNA profiles in $15 E 2 A^{-}$and $15 E 2 A^{+}$ gastric MALT lymphomas are shown in Table 1 and Figure 4. MiR-28-5p and miR-93 were expressed at a median dCT of around -2 or -3 . All the other miRNAs were expressed at a median dCT between -5 and -9 .

Except for miR-223, the differences in median dCTs between the $E 2 A^{-}$and $E 2 A^{+}$groups were all less than one cycle. For miR-223, the median dCT was -9.3 in the $E 2 A^{-}$group and -5.5 in the $E 2 A^{+}$ group $(P=0.001$; Table 1$)$. The stronger expression of the memory B-cell-associated miR-223 distinguished $E 2 A^{+}$from $E 2 A^{-}$gastric MALT lymphomas.

The higher miR-223 level in the $E 2 A^{+}$group could be due to increased granulocytes or histiocytes, two cell types that also express miR-223. Immunohistochemical stain for $C D 68$, a marker for

Table 1 Comparisons between $E 2 A^{-}$and $E 2 A^{+}$gastric MALT lymphomas

\begin{tabular}{lccc}
\hline & $E 2 A^{-}$ & $E 2 A^{+}$ & $\mathrm{P}$ \\
\hline$N$ & 25 & 25 & \\
Age & 59 & 57 & 0.66 \\
Sex & $10: 15$ & $11: 14$ & 1.00 \\
Resistant to antibiotic & $32 \%(8 / 25)$ & $67 \%(16 / 24)$ & 0.03 \\
treatment & & & \\
t(11;18) & $28 \%(7 / 25)$ & $32 \%(8 / 25)$ & 1.00 \\
MUM1 ${ }^{+}$plasmacytoid cells & $48 \%(12 / 25)$ & $16 \%(4 / 25)$ & 0.03 \\
Perigastric lymph node & $11 \%(2 / 19)$ & $55 \%(12 / 22)$ & 0.004 \\
involvement & & & \\
Somatic hypermutations & 18 & 29 & 0.31 \\
Intraclonal variations & 0.3 & 0.4 & 0.77 \\
R/S ratio, framework & 2.0 & 1.9 & 0.85 \\
R/S ratio, complementarity & 2.9 & 2.9 & 0.88 \\
determining & & & \\
dCT miR-28-5p & -3.5 & -3.2 & 0.21 \\
dCT miR-93 & -2.4 & -3.1 & 0.87 \\
dCT miR-17-5p & -5.8 & -5.0 & 0.33 \\
dCT miR-20b & -9.1 & -8.4 & 0.09 \\
dCT miR-181b & -4.9 & -5.6 & 0.95 \\
dCT miR-223 & -9.3 & -5.5 & 0.001 \\
& & & \\
\end{tabular}

From top to bottom, the rows are case numbers, median age of onset in years, male to female ratio, percentages of cases resistant to Helicobacter pylori eradication therapy, with $\mathrm{t}(11 ; 18)$, with plasmacytoid differentiation, or with perigastric lymph node involvement, median number of somatic hypermutations, or intraclonal variations, median ratio of replacement to silent mutations in the framework or complementarity determining regions, and median dCTs for miRNAs. Somatic hypermutations were measured in $10 \mathrm{E} 2 \mathrm{~A}^{-}$and $10 \mathrm{E} 2 \mathrm{~A}^{+}$ cases, and miRNAs were measured in $15 E 2 A^{-}$and $15 E 2 A^{+}$cases. histiocytes, showed about $5-10 \% \mathrm{CD}^{+} 8^{+}$cells in 10 $E 2 A^{-}$and $10 \mathrm{E}^{2} \mathrm{~A}^{+}$cases. As the difference in the dCT of miR-223, $-9.3 \mathrm{vs}-5.5$, was about four cycles, the difference in miR-223 expression should be about 10-20-fold, and was unlikely caused by increased histiocytes alone in the $E 2 A^{+}$group.

\section{Hierarchical Clustering Confirmed Association Between E2A and miR-223}

To confirm the association between $E 2 A$ expression and higher miR-223 levels, hierarchical clustering with both E2A and the whole panel of six miRNAs on 30 gastric MALT lymphomas was performed with Genesis. ${ }^{29}$ Two subtypes of gastric MALT lymphomas, including 17 type I and 13 type II gastric MALT lymphomas, were identified (Figure 4d). The expressions for all of the six miRNAs were higher in type II gastric MALT lymphoma, although only miR223 was significantly different (Table 2). In addition, type II gastric MALT lymphoma was associated with E2A expression (2/17 or $12 \%$ in type I vs $13 / 13$ or $100 \%$ in type II, $P=<0.001$; Table 2 ).

\section{E2A $^{+}$Gastric MALT Lymphomas Were More Likely to Involve the Perigastric Lymph Nodes and More Resistant to H. Pylori Eradication Therapy}

In this study, we excluded cases that had involvement of abdominal lymph nodes when evaluated by abdominal computed tomography ( $\mathrm{II}_{\mathrm{E} 2}$ or beyond). By definition, these gastric MALT lymphomas were in either stage $\mathrm{I}_{\mathrm{E}}$ or $\mathrm{II}_{\mathrm{E} 1}$. Endoscopic ultrasonography was performed in 41 cases to separate cases limited to the stomach (stage $\mathrm{I}_{\mathrm{E}}$ ) from cases extending to the perigastric lymph nodes (stage $\mathrm{II}_{\mathrm{E} 1}$ ).

Perigastric lymph node involvement was noted in 2 of $19 E 2 A^{-}$cases, and in 12 of $22 E 2 A^{+}$cases $(11 \%$ vs $55 \%, P=0.004$; Table 1). This showed that $E 2 A^{+}$ gastric MALT lymphomas were more likely to involve the perigastric lymph nodes.

Consistent with the correlation between $E 2 A$ expression and a more advanced stage, the $E 2 A^{+}$ subtype was more resistant to $H$. pylori eradication therapy. Table 1 showed that 8 out of $25 \mathrm{E}^{2} \mathrm{~A}^{-}$gastric

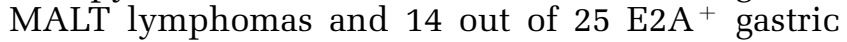
MALT lymphomas were resistant to $H$. pylori eradication therapy ( $32 \%$ vs $56 \%, P=0.03)$.

\section{Discussion}

Aberrant differentiation of lymphomas may cause problems in lineage assignment, but may also be used as a diagnostic tool. Even the best B-cell markers, such as $C D 79 a$ and $C D 20$, have been identified on rare cases of peripheral T-cell lymphomas. ${ }^{30}$ Aberrant expression of $C D 5$, a T-cell marker, is a diagnostic feature of chronic lymphocytic leukemia and mantle cell lymphoma. Other T-cell 


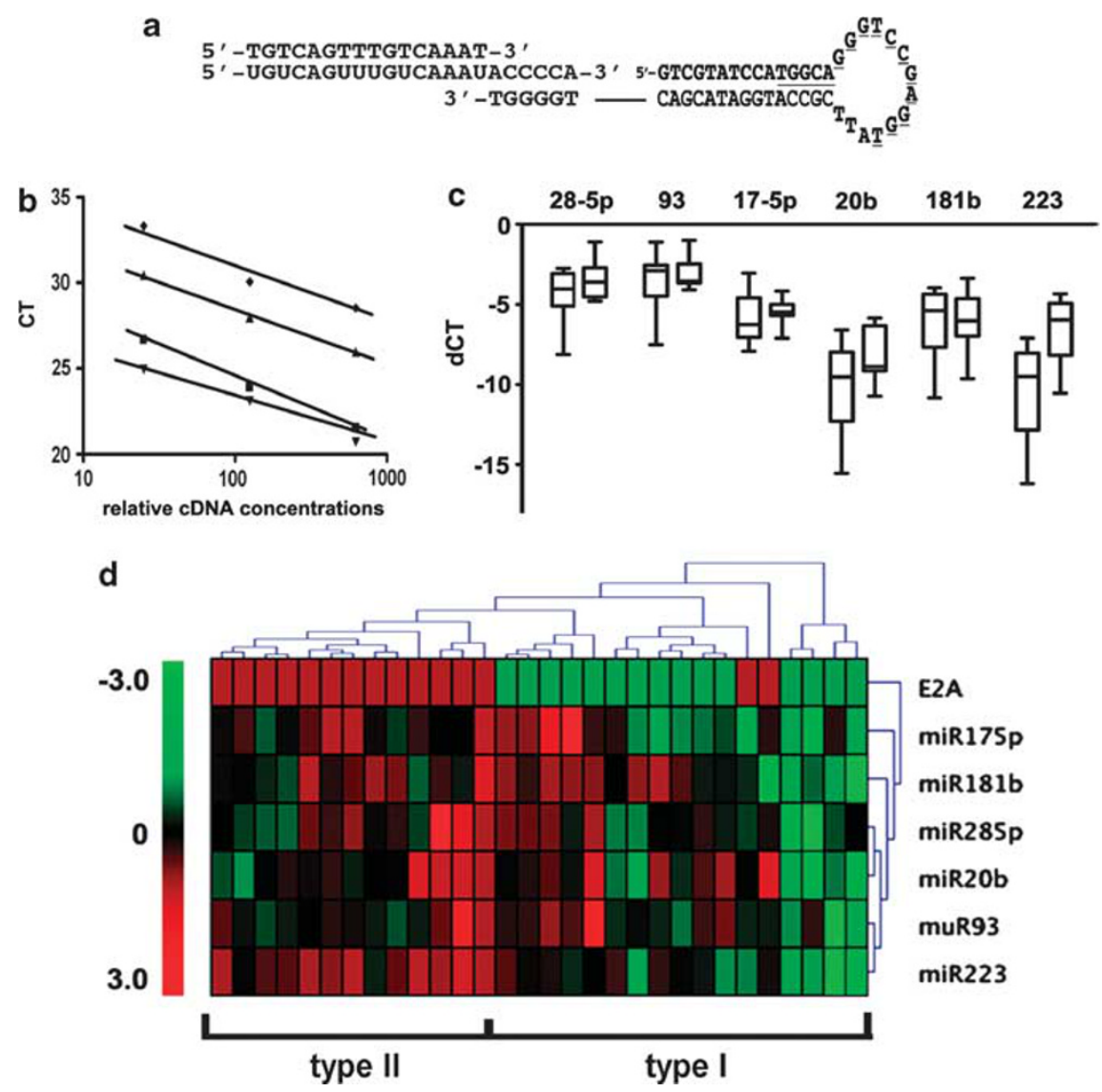

Figure $4 \mathrm{E} \mathrm{A}^{+}$gastric MALT lymphomas had higher levels of the memory B-cell-associated miR-223 than $E 2 A^{-}$gastric MALT lymphomas did. (a) Real-time RT-PCR based on a stem-loop approach is shown schematically. The RT primer has a stem-loop structure to prevent annealing to immature miRNAs, and had six nucleotides at the $3^{\prime}$ end to base pair with mature miRNAs. For PCR, the forward primer is shown above the sequence of $m i R-223$, and the universal reverse primer is underlined to show its overlap with the RT primer. (b) Representative examples of real-time RT-PCRs for miR-223 are shown. An E2A- gastric MALT lymphoma with a dCT of -7.5 and an $E 2 A^{+}$gastric MALT lymphoma with a dCT of -4.1 are shown; $x$ axis: relative amounts of input cDNAs; $y$ axis: CT. From top, the linear regression lines are for miR-223 of an $E 2 A^{-}$gastric MALT lymphoma, miR-223 of an $E 2 A^{+}$gastric MALT lymphoma, $U 6$ of an $E 2 A^{+}$ gastric MALT lymphoma, and U6 of an $E 2 A^{-}$gastric MALT lymphoma. (c) The expression profiles of six miRNAs are summarized in a box-and-whisker plot; $x$ axis: microRNA; $y$ axis: dCT. For each microRNA, the $E 2 A^{-}$group is on the left and the $E 2 A^{+}$group is on the right. Note that the median dCT of $m i R-223$ was -9.3 for the $E 2 A^{-}$group and -5.5 for the $E 2 A^{+}$group $(P=0.001)$. (d) The patterns of $E 2 A$ and six miRNAs were used for hierarchical clustering of 30 gastric MALT lymphomas. Each row is a case of gastric MALT lymphoma, and each column represents $E 2 A$ or an miRNA. The dCTs were normalized to z-scores. Red rectangles represent z-scores above the averages or expression of E2A. Note that the 17 type I gastric MALT lymphomas had lower E2A and miRNA expression, and the 13 type II gastric MALT lymphomas had higher E2A and miRNA expressions.

Table 2 Comparisons between type I and type II gastric MALT lymphomas

\begin{tabular}{lccc}
\hline & Type $I$ & Type II & $\mathrm{P}$ \\
\hline$N$ & 17 & 13 & \\
Age & 61 & 49 & 0.27 \\
Sex (M:F) & $7: 10$ & $6: 7$ & 1.00 \\
E2A & $12 \%(2 / 17)$ & $100 \%(13 / 13)$ & $<0.001$ \\
dCT miR-28-5p & -3.6 & -3.0 & 0.14 \\
dCT miR-93 & -3.0 & -2.8 & 0.53 \\
dCT miR-17-5p & -5.8 & -5.0 & 0.14 \\
dCT miR-20b & -8.9 & -8.4 & 0.26 \\
dCT miR-181b & -5.8 & -5.1 & 0.43 \\
dCT miR-223 & -9.0 & -5.0 & $<0.001$ \\
\end{tabular}

Hierarchical clustering was performed on $15 E 2 A^{+}$and $15 E 2 A^{-}$cases. Note that type I had lower expression of $E 2 A$ and $m i R-223$, whereas type II had higher expression of E2A and miR-223. See Table 1 for abbreviations. antigens, such as $C D 2, C D 7, C D 4$, and $C D 8$, have all been detected in B-cell lymphomas. ${ }^{31}$

B-cell lymphomas are classified by assignment to their normal developmental counterparts. ${ }^{32}$ However, aberrant differentiation among B-cell lymphomas is well documented. Follicular lymphoma might exhibit marginal-zone differentiation, ${ }^{6,7}$ and mantle cell lymphoma could express markers of germinal-center B cells, such as BCL6 or CD10, ${ }^{33,34}$ or could resemble marginal-zone B-cell lymphoma morphologically. ${ }^{35}$ In contrast, data on the aberrant follicular differentiation of marginal-zone B-cell lymphoma are limited. ${ }^{36}$

According to the previous reports, Malt lymphomas had about 15 somatic hypermutations and 0.92 intraclonal variations per immunoglobulin heavy chain variable region, ${ }^{37}$ and follicular lymphoma, 
the archetypical lymphoma with somatic hypermutations and intraclonal variations, had about 29 somatic hypermutations and 2.6 intraclonal variations per immunoglobulin heavy chain variable region. ${ }^{38}$ These reports and our findings showed that most gastric MALT lymphomas had somatic hypermutations without intraclonal variations, consistent with an origin from memory B cells. With this result, the expression of E2A in gastric MALT lymphoma is best regarded as aberrant follicular differentiation.

Most reports on aberrant differentiation were focused on the expression of surface antigens, even though transcription factors are critical in lineage commitment. Among the transcription factors, gastric MALT lymphomas were $B C L 6^{-} / A I D^{-} / T C L 1^{-}$. In addition, gastric MALT lymphomas had somatic hypermutations without intraclonal variations, consistent with an origin of memory B cells. Therefore, the expression of E2A in a subtype of gastric MALT lymphoma was indicative of aberrant follicular differentiation.

$E 2 A$ is critical in early B lymphopoiesis and in the development of germinal-center B cells. ${ }^{39}$ E2A may stimulate somatic hypermutations. ${ }^{10,11}$ In addition, down-regulation of $E 2 A$ is correlated with a reciprocal up-regulation of MUM1, which is a transcription factor required for plasma cell differentiation. ${ }^{12}$ We found that $E 2 A^{-}$and $E 2 A^{+}$groups are similar in the patterns of somatic hypermutations, but the $E 2 A$ + group had less $M U M 1^{+}$plasmacytoid cells. Thus, the expression of E2A in gastric MALT lymphoma does not affect somatic hypermutations, but appears to block plasma cell differentiation. Interestingly, the $\mathrm{E} \mathrm{A}^{+}$group also had a higher expression level of miR-223, which was a memory B-cell-associated miRNA. This raised the possibility that a block in plasma cell differentiation might force the lymphoma cells to differentiate into memory B cells.

MiRNAs are endogenous RNAs of about 19-23 nucleotides. They regulate the translation of mRNAs and control the development of various organ systems. In the B-lineage, distinct patterns of miRNAs are associated with discrete developmental stages, and mature B-cell subsets, such as germinalcenter B cells and memory B cells, could be distinguished by their miRNA profiles. ${ }^{21,22}$

Four groups have proposed panels of 39, 21, 16, or 6 miRNAs to distinguish germinal-center B cells from memory B cells, ${ }^{21,22,40,41}$ with substantial overlaps among these panels. Twelve of the second panel, 13 from the third panel, and all 6 from the fourth panel were also included in the first panel. With this observation, the smallest panel of six miRNAs was used to separate $E 2 A^{-}$from $E 2 A^{+}$ gastric MALT lymphomas. We found that the $E 2 A^{-}$ group and the $E 2 A^{+}$group had quite similar expression of all miRNAs, except for the memory B-cell-associated miR-223. For miR-28-5p, miR-93, miR-17-5p, miR-20b, and miR-181b, the differences in the median dCTs were all less than one cycle.
Only for miR-223 did the difference in the median dCT of four cycles reach a significant $P$-value of $<0.001$.

MiR-223 is expressed at a higher level in both naive and memory $B$ cells than in germinal-center B cells. As gastric MALT lymphoma had somatic hypermutations, the higher level of miR-223 in $\mathrm{E} \mathrm{A}^{+}$gastric MALT lymphoma reflects mostly likely an origin from memory B cells. However, malignant B cells do not necessarily follow an orderly differentiation and the higher miR-223 expression might reflect aberrant differentiation toward naive B cells too.

MiRNA profiles of diffuse large B-cell lymphoma, follicular lymphoma, chronic lymphocytic lymphoma, and mantle-cell lymphoma have been reported, ${ }^{42-44}$ but reports on the miRNA profiles of gastric MALT lymphoma appear to be limited. With a small set of only six miRNAs, we identified correlations between miR-223 and E2A expression. This result seemed to support that a relatively small set of miRNAs accounts for most of the differences in miRNA profiles between cell lineages and tissues. ${ }^{45}$

In conclusion, we have investigated aberrant follicular differentiation in 50 gastric MALT lymphomas. All gastric MALT lymphomas have undergone somatic hypermutations with low intraclonal variations, consistent with a derivation from memory $\mathrm{B}$ cells of the marginal zone, but the expression of $E 2 A$ in $E 2 A^{+}$gastric MALT lymphomas suggests aberrant follicular differentiation. Aberrant follicular differentiation could be used for distinguishing $E 2 A^{+}$ gastric MALT lymphoma from chronic gastritis, two entities sometimes indistinguishable even by clonality tests. $\mathrm{E} \mathrm{A}^{+}$gastric MALT lymphoma had less plasmacytoid infiltrates, which was probably because of a block in plasma cell differentiation by E2A, a higher expression of miR-223, and a tendency to involve perigastric lymph nodes. Further studies would be necessary that would reveal the underlying mechanisms for these associations.

\section{Acknowledgements}

This project was supported by Grants NSC 95-2321B-002-023, NSC 96-2321-B-002-014, NSC 97-2321-B002-009, and NSC 97-2628-B-002-045-MY3 to CWL. We thank the National Taiwan University Center for Genomic Medicine for bioinformatics and technical support.

\section{Disclosure/conflict of interest}

The authors declare no conflict of interest.

\section{References}

1 Suarez F, Lortholary O, Hermine O, et al. Infectionassociated lymphomas derived from marginal zone B cells: a model of antigen-driven lymphoproliferation. Blood 2006;107:3034-3044. 
2 Wotherspoon AC, Doglioni C, Diss TC, et al. Regression of primary low-grade B-cell gastric lymphoma of mucosa-associated lymphoid tissue type after eradication of Helicobacter pylori. Lancet 1993;342:575-577.

3 Liu H, Ruskon-Fourmestraux A, Lavergne-Slove A, et al. Resistance of $\mathrm{t}(11 ; 18)$ positive gastric mucosaassociated lymphoid tissue lymphoma to Helicobacter pylori eradication therapy. Lancet 2001;357:39-40.

4 Yeh KH, Kuo SH, Chen LT, et al. Nuclear expression of BCL10 or nuclear factor kappa B helps predict Helicobacter pylori-independent status of low-grade gastric mucosa-associated lymphoid tissue lymphomas with or without $\mathrm{t}(11 ; 18)(\mathrm{q} 21 ; \mathrm{q} 21)$. Blood 2005;106: 1037-1041.

5 Swerdlow SH, Campo E, Harris NL, et al. Tumours of Haematopoietic and Lymphoid Tissues, WHO Classication of Tumours. IARC Press: Lyon, 2008.

6 Torlakovic EE, Aamot HV, Heim S. A marginal zone phenotype in follicular lymphoma with $\mathrm{t}(14 ; 18)$ is associated with secondary cytogenetic aberrations typical of marginal zone lymphoma. J Pathol 2006; 209:258-264.

7 Jourdan F, Molina TJ, Le Tourneau A, et al. Florid marginal zone differentiation in follicular lymphoma mimicking marginal zone lymphoma of MALT type in the lung. Histopathology 2006;49:426-429.

8 Roulland S, Suarez F, Hermine O, et al. Pathophysiological aspects of memory B-cell development. Trends Immunol 2008;29:25-33.

9 Toellner KM, Jenkinson WE, Taylor DR, et al. Lowlevel hypermutation in $\mathrm{T}$ cell-independent germinal centers compared with high mutation rates associated with T cell-dependent germinal centers. J Exp Med 2002;195:383-389.

10 Quong MW, Martensson A, Langerak AW, et al. Receptor editing and marginal zone B cell development are regulated by the helix-loop-helix protein, E2A. J Exp Med 2004;199:1101-1112.

11 Schoetz U, Cervelli M, Wang YD, et al. E2A expression stimulates Ig hypermutation. J Immunol 2006;177: 395-400.

12 Hauser J, Verma-Gaur J, Wallenius A, et al. Initiation of antigen receptor-dependent differentiation into plasma cells by calmodulin inhibition of E2A. J Immunol 2009;183:1179-1187.

13 Ranuncolo SM, Polo JM, Dierov J, et al. Bcl-6 mediates the germinal center B cell phenotype and lymphomagenesis through transcriptional repression of the DNAdamage sensor ATR. Nat Immunol 2007;8:705-714.

14 Greeve J, Philipsen A, Krause K, et al. Expression of activation-induced cytidine deaminase in human B-cell non-Hodgkin lymphomas. Blood 2003;101: 3574-3580.

15 Pasqualucci L, Guglielmino R, Houldsworth J, et al. Expression of the AID protein in normal and neoplastic B cells. Blood 2004;104:3318-3325.

16 Said JW, Hoyer KK, French SW, et al. TCL1 oncogene expression in B cell subsets from lymphoid hyperplasia and distinct classes of B cell lymphoma. Lab Invest 2001;81:555-564.

17 Herling M, Patel KA, Hsi ED, et al. TCL1 in B-cell tumors retains its normal b-cell pattern of regulation and is a marker of differentiation stage. Am J Surg Pathol 2007;31:1123-1129.

18 Dunn-Walters D, Thiede C, Alpen B. Somatic hypermutation and B-cell lymphoma. Philos Trans R Soc Lond B Biol Sci 2001;356:73-82.
19 Qin Y, Greiner A, Hallas C, et al. Intraclonal offspring expansion of gastric low-grade MALT-type lymphoma: evidence for the role of antigen-driven high-affinity mutation in lymphomagenesis. Lab Invest 1997;76: 477-485.

20 Nardini E, Rizzi S, Ménard S, et al. Molecular phenotype distinguishes two subsets of gastric lowgrade mucosa-associated lymphoid tissue lymphomas. Lab Invest 2002;82:535-541.

21 Malumbres R, Sarosiek KA, Cubedo E, et al. Differentiation stage-specific expression of microRNAs in B lymphocytes and diffuse large B-cell lymphomas. Blood 2009;113:3754-3764.

22 Zhang J, Jima DD, Jacobs C, et al. Patterns of microRNA expression characterize stages of human B-cell differentiation. Blood 2009;113:4586-4594.

23 Hummel M, Oeschger S, Barth TF, et al. Wotherspoon criteria combined with B cell clonality analysis by advanced polymerase chain reaction technology discriminates covert gastric marginal zone lymphoma from chronic gastritis. Gut 2006;55:782-787.

24 Aubin J, Davi F, Nguyen-Salomon F, et al. Description of a novel FR1 IgH PCR strategy and its comparison with three other strategies for the detection of clonality in B cell malignancies. Leukemia 1995;9:471-479.

25 Giudicelli V, Chaume D, Lefranc MP. IMGT/V-QUEST, an integrated software program for immunoglobulin and $\mathrm{T}$ cell receptor $\mathrm{V}-\mathrm{J}$ and $\mathrm{V}-\mathrm{D}-\mathrm{J}$ rearrangement analysis. Nucleic Acids Res 2004;32:W435-W440.

26 Chang B, Casali P. The CDR1 sequences of a major proportion of human germline Ig $\mathrm{VH}$ genes are inherently susceptible to amino acid replacement. Immunol Today 1994;15:367-373.

27 Bose B, Sinha S. Problems in using statistical analysis of replacement and silent mutations in antibody genes for determining antigen-driven affinity selection. Immunology 2005;116:172-183.

28 Chen C, Ridzon DA, Broomer AJ, et al. Real-time quantification of microRNAs by stem-loop RT-PCR. Nucleic Acids Res 2005;33:e179.

29 Stum A, Quackenbush J, Tranjanoski A. Genesis: cluster analysis of microarray data. Bioinformatics 2002;18:207-208.

30 Yao X, Teruya-Feldstein J, Raffeld M, et al. Peripheral T-cell lymphoma with aberrant expression of CD79a and CD20: a diagnostic pitfall. Mod Pathol 2001;14:105-110.

31 Kaleem Z, White G, Zutter MM. Aberrant expression of T-cell-associated antigens on B-cell non-Hodgkin lymphomas. Am J Clin Pathol 2001;115:396-403.

32 Sagaert X, Sprangers B, De Wolf-Peeters C. The dynamics of the B follicle: understanding the normal counterpart of B-cell-derived malignancies. Leukemia 2007;21:1378-1386.

33 Camacho FI, García JF, Cigudosa JC, et al. Aberrant Bcl6 protein expression in mantle cell lymphoma. Am J Surg Pathol 2004;8:1051-1056.

34 Zanetto U, Dong H, Huang Y, et al. Mantle cell lymphoma with aberrant expression of CD10. Histopathology 2008;53:20-29.

35 Mansoor A, Akbari M, Auer I, et al. Cyclin D1 and $\mathrm{t}(11 ; 14)$-positive B-cell neoplasms resembling marginal zone B-cell lymphoma: a morphological variant of mantle cell lymphoma. Hum Pathol 2007;38:797-802.

36 Wenzel C, Dieckmann K, Fiebiger W, et al. CD5 expression in a lymphoma of the mucosa-associated lymphoid tissue (MALT)-type as a marker for early 
dissemination and aggressive clinical behaviour. Leuk Lymphoma 2001;42:823-829.

37 Bende RJ, Aarts WM, Riedl RG, et al. Among B cell non-Hodgkin's lymphomas, MALT lymphomas express a unique antibody repertoire with frequent rheumatoid factor reactivity. J Exp Med 2005;201: 1229-1241.

38 Aarts WM, Bende RJ, Steenbergen EJ, et al. Variable heavy chain gene analysis of follicular lymphomas: correlation between heavy chain isotype expression and somatic mutation load. Blood 2000;95:2922-2929.

39 Kwon K, Hutter C, Sun Q, et al. Instructive role of the transcription factor E2A in early B lymphopoiesis and germinal center $\mathrm{B}$ cell development. Immunity 2008;28:751-762.

40 Basso K, Sumazin P, Morozov P, et al. Identification of the human mature B cell miRNome. Immunity 2009; 30:744-752.
41 Tan LP, Wang M, Robertus JL, et al. miRNA profiling of B-cell subsets: specific miRNA profile for germinal center B cells with variation between centroblasts and centrocytes. Lab Invest 2009;89:708-716.

42 Mraz M, Pospisilova S, Malinova K, et al. MicroRNAs in chronic lymphocytic leukemia pathogenesis and disease subtypes. Leuk Lymphoma 2009;50:506-509.

43 Navarro A, Beà S, Fernández V, et al. MicroRNA expression, chromosomal alterations, and immunoglobulin variable heavy chain hypermutations in Mantle cell lymphomas. Cancer Res 2009;69:7071-7078.

44 Roehle A, Hoefig KP, Repsilber D, et al. MicroRNA signatures characterize diffuse large B-cell lymphomas and follicular lymphomas. Br J Haematol 2008;142: 732-744.

45 Landgraf P, Rusu M, Sheridan R, et al. A mammalian microRNA expression atlas based on small RNA library sequencing. Cell 2007;129:1401-1414. 